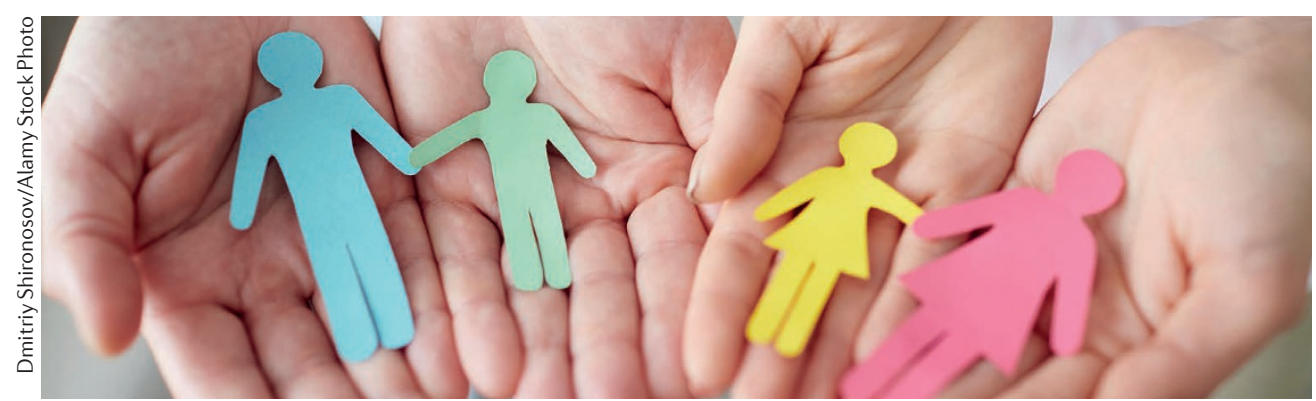

INFLAMMATORY MYOPATHIES

\title{
Adult and paediatric experts unite for new response criteria
}

An international body of experts has developed standardized clinical response criteria to measure minimal, moderate and major improvements in adult patients with dermatomyositis or polymyositis and in children with juvenile dermatomyositis. The new response criteria, published simultaneously in Arthritis \& Rheumatology and Annals of the Rheumatic Diseases, comprehensively cover both adult and paediatric disease using one set of defined core set measures. "The same definitions can be applied to adult and juvenile myositis populations, and clinical trials can be compared with each other," states Rohit Aggarwal, corresponding author for the adult criteria. "Moreover, combined adult and juvenile clinical trials are possible using the newly developed response criteria," he continues.

Previously, core set measures and definitions of improvement had been created and validated independently by the International Myositis Assessment and Clinical Studies Group (IMACS) and by the Paediatric Rheumatology International Trials Organisation (PRINTO). The paediatric criteria (PRINTO) were provisionally approved by the ACR and EULAR for use in patients with juvenile dermatomyositis. "Since the two sets of core set measures displayed some differences, the core set measures were weighted equally and there were no criteria for moderate or major improvement, IMACS and PRINTO, supported by the ACR and EULAR, thought it was time to revise the prior criteria in order to have standardized improvement criteria for all levels of improvement for use in future clinical trials," explains Nicolino Ruperto, corresponding author for the juvenile criteria.

Using a variety of data-driven methodologies, an international group of experts representing IMACS and PRINTO developed a list of candidate definitions for response criteria. These criteria were initially validated using real-life data from patients that had been scored for minimal, moderate or major improvement by expert consensus. The candidate definitions were further validated using data from two clinical trials before being discussed at a consensus meeting of experts in dermatology, neurology and rheumatology. Paediatric and adult experts worked separately before coming together to agree on the final response criteria and thresholds for defining minimal, moderate and major improvement.

The group of experts decided on a conjoint analysis-based continuous model with a continuous assessment score of $0-100$ with thresholds for improvement in children of $\geq 30 / 100$ (minimal), $\geq 45 / 100$ (moderate) and $\geq 70 / 100$ (major), and thresholds for improvement in adults of $\geq 20 / 100$ (minimal), $\geq 40 / 100$ (moderate) and $\geq 60 / 100$ (major). "These differences in the threshold for improvement were likely due to the increased ability of children to display a greater level of improvement when compared with adult patients," points out Ruperto. "These response criteria, endorsed by the ACR and EULAR, should be highly acceptable and widely used given that they were developed with a consensus and data-driven approach involving many myositis experts from around the world," he enthuses.

"For me, the most significant finding is the ability of this definition to be used as a continuous outcome measure as well as a categorical outcome," says Aggarwal. "The total improvement score (0-100 scale) is associated with the degree of improvement in various core set measures, so the higher the degree of improvement in a larger number of core set measures, the higher the total improvement score," he explains.

Looking towards the future, the experts involved in creating these criteria hope that endorsement by the ACR and EULAR will help to promote the use of these new criteria in clinical trials. They are also working on ways to encourage the use of these criteira in both adult and paediatric studies. "We are developing a web-based tool for easy calculation of clinical improvement using the new response criteria by researchers and clinicians around the world," says Aggarwal.

\section{Joanna Collison}

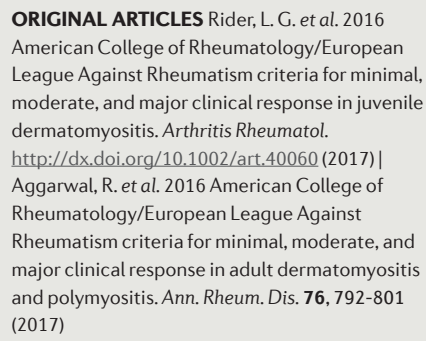

